EPJ Web of Conferences 52, 09001 (2013)

DOI: $10.1051 /$ epjconf/20135209001

(C) Owned by the authors, published by EDP Sciences, 2013

\title{
Uncertainties on low-x parton densities and the corresponding high-energy neutrino cross sections
}

\author{
Amanda Cooper-Sarkar ${ }^{1, a}$ \\ ${ }^{1}$ University of Oxford, Great Britain
}

\begin{abstract}
A brief review of how parton densities are determined within the conventional DGLAP formalism of NLO QCD will be given with particular reference to the low-x region. Predictions for high energy neutrino and antineutrino charged and neutral current cross-sections using several different modern PDF fits will be compared. PDF uncertainties from model assumptions and parametrization bias are considered in addition to the experimental uncertainties. Particular attention is paid to assumptions and biases which could signal the need for extension of the conventional formalism to include effects such as $\ln (1 / x)$ resummation or non-linear effects of high gluon density.

See the published paper [1].
\end{abstract}

\section{References}

[1] Cooper-Sarkar, A. et al, arXiv:1106.3723 [hep-ph], JHEP 1108(2011)042, August 2011.

Slides

The slides of the talk can be found on the website of the symposium ISVHECRI 2012:

https://indico.desy.de/conferenceOtherViews.py?view=standard\&confId=4594

a a.cooper-sarkar1@physics.ox.ac.uk 